\title{
A Study on the Consumer Behavior of Online Ordering Platforms and Its Influencing Factors
}

\author{
Wenbo Chen ${ }^{1, a}$, Weilun Huang ${ }^{1, b,{ }^{*}}$ \\ ${ }^{1}$ Wenzhou Business School, Zhejiang, China \\ a970047636@qq.com, bhuangwl@wzbc.edu.cn \\ ${ }^{*}$ Corresponding author
}

\begin{abstract}
Keywords: Consumer Behavior, Online Ordering Platforms, Influencing factors.
\end{abstract}
\begin{abstract}
The purpose of this paper is to use a literature review and questionnaire analysis to explore the consumer behavior of online ordering platforms (OOPs) and its influencing factors. The results showed that consumers' average frequency of OOPs (Frequency) was 5.5 times a week, and consumers' monthly average amount of OOPs (Amount) was about RMB 360. Consumers' trust (CT), risk (CR), and experience (CE) on OOPs, and consumers' individual variables were the influencing factors on consumer behavior of OOPs. This paper suggests that governments should strengthen the supervision of food safety, rectify fake online ordering advertisement, standardize the behavior of food delivery personnel, and protect consumers' personal information. This paper also suggests that the OOPs should review the hygiene status of the sellers, carry out business in multiple fields, improve the efficiency of delivery, and establish a refined ordering system.
\end{abstract}

\section{Introduction}

Nowadays, the online catering industry is developing rapidly. More and more consumers choose to meet their daily dietary needs by using online ordering platforms (OOPs). consumer behavior of OOPs has been received attention from governments and industries, but there is only a little literature concentrating on this topic. This paper thus aims to explore the consumer behavior of the OOPs and its influencing factors. Ai-Media Consulting (2018) found that the market scale of China's OOP grew from RMB 21.68 billion in 2011 to RMB 243 billion in 2018, with an increase of approximately 11 times. Moreover, China's OOPs users grew from 63 million in 2011 to 355 million in 2018, with a growth of 6 times. On November 6th, 2017, the National Food and Drug Administration of China promulgated regulations to regulate food safety of the internet catering industry (National Food and Drug Administration of China, 2017).

According to the review of literature, online ordering is an important research topic, but there are still few studies discussing the behavior and influencing factors of OOPs. This paper sorted out the existing literature in China and found that research with online ordering in the title started in 1990. In 2016, 81 papers were published in this field, which was the most significant yearly number. There were 977 studies published until June 15th, 2019. However, there were too many papers, so this paper chose to focus on the literature of OOPs in the title after 2013, and therefore 43 articles were chosen. The research field mainly belongs to the field of the guidance of the Internet catering industry, and the focus is on exploring online ordering, food safety, third-party platform supervision, and OOPs. This paper aims to explore the consumer behavior and influencing factors of the OOPs, to supplement the inadequacies of literature, and to provide advice to individuals, companies, and governments.

Form the studies of previous literature; this paper defines the variables of consumer behavior on OOPs are consumers' average frequency of OOPs in a week (Frequency), consumers' average amount of OOPs (Amount), RMB and the ordering meal type of OOPs (TYPE). The TYPE is Chinese simple snacks(CS), local snacks (LS), dessert and drinks (DD), and foreign dishes (FD). For example, He (2016) suggests that consumers in Beijing are more inclined to choose CS lunches when they are ordering online, and Han (2016) believes that the market scale of the online catering industry in 2014 
was RMB 97.5 billion and it was expected to break through RMB 200 billion in 2017. Yang (2018) contends that the Frequency in Lanzhou is five times a week.

According to the literature review, many factors are influencing the consumer behavior of OOPs. This paper sets its influencing factors are Consumers' trust (CT), risk (CR), and experience (CE) on OOPs. Chen (2017) believes that consumers' sanitary conditions, food and beverage tastes, and the delivery time and food prices of OOPs are the factors may be influencing the consumer behavior of OOPs. Chen et al. (2018) believes influencing factors on consumer behavior of OOPs mainly includes consumers' perceived practicability, concession, risk, and popularity.

The variables of CT in this paper are defined as the reliability of OOPs, consumers' recommendation of OOPs, and consumers' confidence of OOPs, based on the study of previous literature. Zhang (2015) believes that the influencing factors of the consumer behavior of OOPs on fresh agricultural products are CT toward the OOPs and consumers' perceived risk. Hong (2017) argues that Food Ordering Service System enhanced the satisfaction of CE and created a more commercial value and social value. Zhang et al. (2018) believes that it is necessary for the companies of OOPs to improve their applications' credibility to gain trust from their customers.

The variables of CR in this paper are defined as OOPs' risks in payment information, registration information, ordering process, family acceptance, and delivery time, according to previous studies. Chen (2018) believes that the influencing factors of Hunan consumer behavior of OOPs are consumers' private risks. Liu (2019) believes that the influencing factors of the Hebei consumer behavior of OOPs are there risks of payment information and individual information. Dharmawirya (2012) finds that consumers spend 5.4 minutes waiting to get their orders on average, during peak hours in lunch time.

The variables of $\mathrm{CE}$ in this paper are defined as the usability of OOPs, the usefulness of OOPs, design of OOPs, timeliness of message push and payment security of OOPs, according to previous studies. Chen et al. (2018) believes that factors of Hunan consumer behavior of OOPs are there perceived usefulness and usability of OOPs. Han (2009) finds that the Mobile Food Ordering System offers many useful features to consumers, including the location of the food store on the map, detailed menus, and previous order lookup. Yang et al. (2012) contends that the usefulness of OOPs has a significant influence on consumers' intentions.

According to the above discussion, the focus of this paper is the consumer behavior of OOPs and its influencing factors, and this paper discusses the impacts of consumers' variables which are their gender (GEN), age (AGE), education (EDU), monthly income (MI) and food expenditure (FE), on the consumer behavior of OOPs and its influencing factors. Wang et al. (2018) considers the influencing factors on Yangzhou consumer behavior of OOPs is there AGE, MI, family population and individual confidence.

In addition to the introduction, the rest of the paper is divided into two parts: the second part is the analysis on the results of the questionnaires, and the third part is conclusion and suggestions.

\section{The analysis of questionnaires}

This questionnaire was released online in China from June 23rd to June 30th, 2019. The number of valid questionnaires was 537. The statistics of consumers' variables are listed as follows: 1 . GEN (\%): $47.7 \%$ were males and $52.3 \%$ were females. 2. AGE: their average age was 27.6 (standard deviation (SD) is 5.9). 3. EDU (\%): $46 \%$ of the respondents were below undergraduate, $54 \%$ were undergraduate and above. 4. MI (RMB): their average monthly income was 4,379.9 (SD is 1,994.4). 5. FE (RMB): their average food expenditure was 1,606.1 ( $\mathrm{SD}$ is 924.6).

The descriptive statistical results of the behavioral variables of consumer behavior of OOPs are listed as follows: the Frequency was 5.5 times (SD is 2.2); the Amount (RMB) was 355.2 (SD is 241.2); in terms of TYPE, FD accounted for 31.8\%, CS accounted for 30.5\%, LS accounted for $22.9 \%$, and DD accounted for $15.6 \%$ of respondents would online ordering.

The descriptive statistical results of the influencing factors on consumer behavior of OOPs are about $70 \%$ of the respondents agreed is influencing factors are CT (approval ratio was 76.0\%), CR 
(63.7\%), and CE (60.3\%). Respondents' ratings in terms of CT on OOPs were the reliability of OOPs (approval ratio was 62.9\%), the consumers' recommendation of OOPs $(57.2 \%)$, and consumers' confidence (42.8\%). Respondents' ratings in terms of CR on OOPs were registration information $(66.2 \%)$, delivery time $(63.1 \%)$, payment information $(36.7 \%)$, acceptance of family, $(33.1 \%)$, and ordering process $(31.7 \%)$. Respondents' ratings in terms of CE on OOPs were payment security $(49.9 \%)$, timeliness of OOPs' message $(48.8 \%)$, design $(45.1 \%)$, usefulness $(44.4 \%)$ and usability $(39.3 \%)$.

In this paper, the variance analysis on the consumer behavior of OOPs and its influencing factors were shown in Table 1. It can be seen from Table 1 that the significant influencing factors of Frequency and Amount were CT, CR, and CE on OOPs. The TYPE that significantly affected by CT was LS. Table 1 also showed that the relationships between Frequency and CT, CR, CE on OOPs were significantly positive, with correlation coefficients at $0.3,0.2$, and 0.5 respectively; the relationships between Amount and CT, CR, CE on OOPs were also significantly positive, with correlation coefficients at $0.4,0.4$, and 0.8 respectively.

Table 1. The variance analysis on the consumer behavior of OOPs and its influencing factors.

\begin{tabular}{ccccccc}
\hline \multirow{2}{*}{$\begin{array}{c}\text { Influencing } \\
\text { factors }\end{array}$} & Frequency & Amount & CS & FD & LS & DD \\
\hline CT & $5.1^{* * *}$ & $5.6^{* * *}$ & 0.80 & 1.2 & $1.6^{*}$ & 0.5 \\
CR & $1.9^{*}$ & $6.2^{* * *}$ & 1.6 & 1.3 & 0.6 & 0.6 \\
$\mathrm{CE}$ & $16.8^{* * *}$ & $145.6^{* * *}$ & 0.9 & 0.5 & 0.6 & 1.0 \\
\hline
\end{tabular}

Note: ${ }^{*} \mathrm{p}<0.05, \quad * * \mathrm{p}<0.01, \quad * * * \mathrm{p}<0.001$.

In order to further understand the results of the questionnaires, this paper explored the impacts of respondents' GEN, AGE, MI, EDU and FE on the consumer behavior of OOPs and its influencing factors (see Table 2). From Table 2, respondents' AGE, MI, EDU, and FE are positively related to Frequency and Amount. Respondents' GEN, MI, and EDU have significant impacts on the TYPE. For example, Male respondents would choose LS of OOPs significantly more than female; similarly, the respondents' with higher MI would choose FD of OOPs significantly more than others; the respondents' with higher EDU would choose LS of OOPs significantly more than others ; the respondents' with lower EDU would choose CS of OOPs significantly more than others .respondents' AGE, MI, EDU, and FE have significant positive impacts on CT,CR,CE of OOPs.

Table 2. The variance analysis independent sample t-test on the impact of respondents individual variables have on the consumer behavior of OOPs and its influencing factors.

\begin{tabular}{|c|c|c|c|c|c|c|c|}
\hline \multirow{8}{*}{$\begin{array}{l}\text { Consumer } \\
\text { behavior of } \\
\text { OOPs }\end{array}$} & & & GEN & Age & MI & EDU & $\mathrm{FE}$ \\
\hline & & & $\mathrm{F}(\mathrm{T})$ & $\mathrm{F}$ & $\mathrm{F}$ & $\mathrm{F}(\mathrm{T})$ & $F$ \\
\hline & \multicolumn{2}{|c|}{ Frequency } & $\begin{array}{c}3.2 \\
(-0.6)\end{array}$ & $12.8 * * *$ & $8.3^{* * *}$ & $\begin{array}{c}2.3 \\
(-4.0 * * *)\end{array}$ & $8.4 * * *$ \\
\hline & \multicolumn{2}{|c|}{ Amount } & $\begin{array}{c}0.9 \\
(0.1)\end{array}$ & $23.0 * * *$ & $13.8 * * *$ & $\begin{array}{c}33.1 * * * \\
(-8.6 * * *)\end{array}$ & $7.3 * * *$ \\
\hline & \multirow{4}{*}{ Type } & $\mathrm{CS}$ & $\begin{array}{c}0.5 \\
(0.3)\end{array}$ & 1.5 & 2.0 & $\begin{array}{l}5.9^{*} \\
(1.2)\end{array}$ & 1.2 \\
\hline & & FD & $\begin{array}{c}2.8 \\
(0.8)\end{array}$ & 1.2 & $2.1^{*}$ & $\begin{array}{c}1.5 \\
(0.6)\end{array}$ & $5.1 * *$ \\
\hline & & LS & $\begin{array}{c}16.1 * * * \\
\left(-2.0^{*}\right)\end{array}$ & 1.7 & 0.9 & $\begin{array}{c}12.9 * * * \\
(-1.8)\end{array}$ & 1.0 \\
\hline & & DD & $\begin{array}{c}1.4 \\
(-0.6)\end{array}$ & 1.7 & 1.9 & $\begin{array}{c}0.5 \\
(-0.4)\end{array}$ & 0.6 \\
\hline $\begin{array}{l}\text { Influencing } \\
\text { factors }\end{array}$ & \multicolumn{2}{|c|}{$\mathrm{CT}$} & $\begin{array}{c}0.7 \\
(-1.1)\end{array}$ & $11.1 * * *$ & $5.6 * * *$ & $\begin{array}{c}0.4 \\
\left(-3.7^{* * *}\right)\end{array}$ & $11.4^{* * *}$ \\
\hline
\end{tabular}




\begin{tabular}{|c|c|c|c|c|c|c|}
\hline \multirow[t]{3}{*}{ Consumer } & & GEN & Age & MI & EDU & FE \\
\hline & CR & $\begin{array}{l}1.0^{*} 10^{\wedge}-2 \\
\left(-1.4^{* * *}\right)\end{array}$ & $10.8^{* * *}$ & $9.9 * * *$ & $\begin{array}{c}1.0^{*} 10^{\wedge}-3 \\
\left(-2.1^{*}\right)\end{array}$ & $6.7 * * *$ \\
\hline & $\mathrm{CE}$ & $\begin{array}{c}0.1 \\
(-0.1)\end{array}$ & $23.0 * * *$ & $10.0 * * *$ & $\begin{array}{c}0.3 \\
\left(-10.3^{* * *}\right)\end{array}$ & $3.3^{*}$ \\
\hline
\end{tabular}

Note: ${ }^{*} \mathrm{p}<0.05, \quad * * \mathrm{p}<0.01, \quad * * * \mathrm{p}<0.001$.

According to above results, this paper suggested:

(1) For the hygienic situation of sellers on OOPs, the enterprise of OOPs should strengthen their hygienic survey and governments should conduct comprehensive supervision on their food safety, and clarify the rules and responsibilities to protect the consumers' health. This paper found the Frequency was 22 times per month, and the Amount was (RMB) 355.2 which was account $8.1 \%$ of their MI and $22.1 \%$ of the their FE, these indicated the consumption of OOPs had become the major dining mode for consumers. Therefore, enterprises of OOPs should set a new supervision mode on the sellers of OOPs which relying on a multi-level, multi-regional take-out information database. Chinese government issued a new food safety law in 2015, but its enforceability was not strong and did not focus on sellers of OOPs.

(2) The enterprises of OOPs should disclose the information consistent with the content of the food hygiene license of OOPs' sellers, and governments should punish the fake advertisement of OOPs. According to the results of this paper, CT was a significant influencing factor in the consumer behavior of OOPs, and $60 \%$ of respondents agree the reliability of OOPs was important. Therefore, the enterprises of OOPs should announce containers, packaging, and equipment used by OOPs' sellers, in order to keep their food safe. And governments should strengthen administrative punishment, penalty and stop webcasting fake advertisements. At present, there are few cases of punishment, so the government should strengthen supervision of OOPs' sellers.

(3) The enterprises of OOPs should use technology of AI, big data, network security, confidentiality system to reduce the consumers' perceived risks of OOPs, and governments should regulate the delivery behavior of food dispatchers. According to the survey results, CR was a significant influencing factor in the consumers' behavior of OOPs. About $60 \%$ of the respondents agreed their registration information and delivery time of OOPs were important. Although the Article 13 of the Measures for the Supervision and Administration of Food Safety in Online Catering Services issued by the Chinese government in 2017 stipulated that OOPs providers of online catering services should provide more training of food safety and strengthen management for food delivery dispatchers; however, the personal and moral reliability of the food delivery dispatchers still need to be reviewed by OOPs to ensure the efficiency of delivery and quality of the meal.

(4) The enterprises of OOPs should use automatically statistics technology and push horizontal alliances with convenience stores to make OOPs more convenience for both consumers and sellers, and governments should ensure the stability and security on the payment of OOPs. According to the survey results, CE was a significant influencing factor in the consumers' behavior of OOPs. The respondents agreed the usability, the usefulness, design, timeliness of message push and payment security of OOPs were important. The sellers of OOPs can carry out business statistics automatically and perform business settlement without manual reconciliation. The OOPs' horizontal alliances with convenience stores could promote convenience for consumers with the variety of goods and the widened price range commodities. Governments should insist the banking system is the leading of OOPs' payment security supervision and management mechanism, and the other government departments should be is auxiliary.

(5) The enterprises of OOPs should implement differentiated marketing strategies based on consumers' gender, age, monthly income, education, and food expenditure. According to the survey results, consumers' gender, age, monthly income, education, and food expenditure would partially significant affect the consumer behavior of OOPs and its influencing factors. Therefore, this paper 
suggests that enterprises of OOPs should use differentiated advertisements and notifications strategies according to gender, age, monthly income, education and food expenditure. For example, the enterprises of OOPs could push information of LS and delivery situation for male consumers, and the information of concessions were for the consumers who are older, with higher MI, higher EDU, or higher FE.and the exemption information of delivery fees were for the consumers with higher MI and FE.

\section{Conclusion and suggestions}

Nowadays, the online ordering platforms have become an important part of the catering industry, and the market scale of online ordering is constantly growing. However, there is no law and regulation regarding online ordering platforms in China, the consumer behavior of online ordering platforms and its influencing factors should be an important research topic for enterprises, governments, and scholars. So the purpose is of this paper to study consumer behavior of online ordering platforms and its influencing factors, and the impact of consumers' individual variables on them.

According to the review of literature and the statistical results of the questionnaires, the consumers' average frequency of online ordering platforms in a week was 5.5 times, and the consumers' average weekly amount of online ordering platforms was RMB 355.2. Consumers' trust, consumers' risk, and consumers' experience were significant influencing factors of online ordering platforms. Consumers' gender, age, monthly income, education and food expenditure could impact consumer behavior of online ordering platforms and influencing factors which are consumers' perceived trust, risk and experience of online ordering platforms.

Based on the above discussion, this paper suggests that governments should conduct the comprehensive supervision system on the food safety, the fake advertisement, delivery behavior of food dispatchers, the stability and security on the payment of online ordering platforms. This paper also suggests that enterprises of online ordering platforms should strengthen their hygienic survey system, disclose the information of their sellers, and formulate a multi-field, fast delivered, and easy-to-use marketing strategies.

The future research direction of this paper is to study more influencing factors on consumer behavior of online ordering platforms, such as consumers' perceived popularity and preference of online ordering platforms. Chen (2018) suggests that perceived consumers' preference and perceived popularity should have a significant positive effect on consumers' online ordering behavior, while consumers' perceived risk has a significant negative correlation with consumers' online ordering behavior.

\section{References}

[1] Yang H., Chen J., Huang X. , and Li C., Food safety analysis of online ordering platform from the perspective of college students, China High-tech Zone, vol. 06, pp. 54-55+57, 2018 .

[2] Han H., Research on college students' online ordering market based on takeaway O2O model take Nanchang City as an example, Shang, vol. 11, pp. 298, 2016.

[3] He Y., Ji A., Li H., Study on the influencing factors of college students' online ordering behavior, Enterprise Economy, vol. 07, pp. 83-89, 2016.

[4] Chen Z., Lu F., Research on the behavior of TAM-based college students using "hungry?" to order meals, Journal of Hunan University of Technology, vol. 32(05), pp. 72-77, 2018.

[5] Zhang Y., Zhang M., Wang Q., Ren Y., Ma S., Ma S., Shao W., Yin S., and Shi Z., Study on purchasing Intention of fresh agricultural products under the $\mathrm{O} 2 \mathrm{O}$ model based on perceived return-perceived risk framework, Soft Science of China, vol. 06, pp. 128-138, 2015.

[6] Liu P., Analysis of the risks and preventive measures of mobile payment in college finance, Modern Marketing (Information Edition), vol. 05, pp. 22, 2019. 
[7] Wang T., Pan X., Gao Z., and Cui B., Study on the perceived risk of consumers' online ordering and its influencing factors, Reform and opening up, vol. 09, pp. 95-97, 2018.

[8] Chen X., and Li Y., Comparative analysis of different online ordering modes for college students, China Market, vol. 07, pp. 109-112, 2017.

[9] Chen J., Yang H., Huang X., and Li C., Current situation and countermeasures of college students' online ordering platform, Legal Exposition, vol. 16, pp. 43+42, 2018.

[10]Dharmawirya M., Oktadiana H., and Adi E., Analysis of expected and actual waiting time in fast food restaurants, Industrial Engineering Letters, vol. 2(5), pp. 8-17, 2012.

[11]Kullkami H., Dascalu S. M., and Harris C. F., Software development aspects of a mobile food ordering system, Software Engineering, pp. 67-72, 2009.

[12]Hong X., Food ordering service system design for Chinese urban commuters based on Internet of things. In: Streitz N., Markopoulos P. (eds) Distributed, Ambient and Pervasive Interactions, Lecture Notes in Computer Science, vol. 10291, Springer, Cham, 2017.

[13]Zhang Y., Wang J., and Wang J., The challenges of online meal ordering platforms: website design and credibility, customers' trust, and food safety. In: Ho A. (eds) Advances in Communication of Design. AHFE 2017, Advances in Intelligent Systems and Computing, vol. 609, Springer, Cham, 2018.

[14] Yang L., Weng T., and He H., Online ordering systems and consumer acceptance - using box lunch order systems as a case study, International Journal of Biometrics, vol. 7(18), 2012. 